\title{
Efeito de bebidas corantes e ácidas na estabilidade da cor do esmalte dentário após clareamento de consultório
}

\author{
Effect of coloring and acidic beverage on color stability of enamel after office bleaching \\ Efecto de bebidas colorantes y ácidas sobre la estabilidad del color del esmalte después \\ del blanqueamiento en consultório dentario
}

Jiovanne Rabelo Neri ${ }^{1,2}$, Lara Rabelo Aragão ${ }^{1}$, Flávia Maria Noronha Nigri ${ }^{1}$, Vanara Florêncio Passos $^{3}$, Lívia de Oliveira Barros ${ }^{1}$, Fernanda Araújo Sampaio ${ }^{1}$, Phillipe Nogueira Barbosa Alencar ${ }^{1 *}$, Juliana Paiva Marques Lima Rolim¹.

\section{RESUMO}

Objetivo: Avaliar a influência de bebida corantes e ácidas na estabilidade de cor do esmalte clareado com peróxido de hidrogênio à $35 \%$. Métodos: Vinte terceiros molares foram seccionados para obter fragmentos de $4 \times 4 \mathrm{~mm}$ e polidos com lixas de granulação 320, 400, 600 e 1200. Os espécimes foram divididos aleatoriamente em 6 grupos $(n=6)$, de acordo com as bebidas: água destilada, refrigerante à base de cola, vinho tinto, açaí, café e cerveja escura. A cor de cada amostra foi avaliada com um espectrofotômetro em 3 momentos: antes do clareamento (baseline), após o clareamento com peróxido de hidrogênio a $35 \%$, durante 45 minutos, e após a imersão em bebidas corantes. A medição das cores foi baseada na escala CIE L*a*b*. Os dados foram analisados com ANOVA e Kruskal-Wallis, as comparações pós-hoc foram testadas como teste de Student-Newman-Keuls e o nível de significância foi de $p<0,05$. Resultados: Não houve diferença estatística entre os valores dos grupos $\Delta \mathrm{E}^{*}$ refrigerante à base de cola, vinho tinto, suco de açaí, café e cerveja preta $(p>0,05)$. Conclusão: Refrigerantes à base de cola, vinho tinto, suco de açaí, café e cerveja escura interferem na estabilidade da cor do esmalte clareado com peróxido de hidrogênio a 35\%.

Palavras-chave: Clareamento dentário, Peróxido de hidrogênio, Esmalte dentário.

\section{ABSTRACT}

Objective: To evaluate the influence of colorants and acids on the color stability of $35 \%$ hydrogen peroxide bleached enamel. Methods: Twenty third molars were sectioned to $4 \times 4 \mathrm{~mm}$ fragments and polished with 320 , 400,600 and 1200 grit sandpaper. The specimens were randomly divided into 6 groups $(n=6)$ according to the colorants: distilled water, cola-based soda, red wine, acai, coffee and dark beer. The color of each sample was evaluated with a spectrophotometer at 3 times: before bleaching (baseline), after bleaching with $35 \%$ hydrogen peroxide for 45 minutes, and after immersion in coloring drinks. The color measurement was based on the CIE L * $a$ * $b$ * scale. Data were analyzed with ANOVA and Kruskal-Wallis, post-hoc comparisons were tested as Student-Newman-Keuls test and significance level was $p<0.05$. Results: There was no statistical difference between the values of the $\Delta \mathrm{E}{ }^{*}$ cola-based soda, red wine, acai juice, coffee and dark beer groups

${ }^{1}$ Centro Universitário Christus (UNICHRISTUS), Fortaleza, CE, Brasil.

*E-mail: drphillipenogueira@hotmail.com

2 Universidade de Fortaleza (UNIFOR), Fortaleza, CE, Brasil.

3 Universidade Federal do Ceará (UFC), Fortaleza, CE, Brasil. 
( $p>0.05)$. Conclusion: Cola-based soda, red wine, acai juice, coffee and dark beer interfere with the color stability of the $35 \%$ hydrogen peroxide bleached enamel.

Key words: Tooth bleaching, Hydrogen peroxide, Dental enamel

\section{RESUMEN}

Objetivo: Evaluar la influencia de los colorantes y las bebidas ácidas en la estabilidad del color del esmalte blanqueado con peróxido de hidrógeno al $35 \%$. Métodos: Se seccionaron veintitrés molares en fragmentos de $4 \times 4 \mathrm{~mm}$ y se pulieron con papel de lija de grano 320,400, 600 y 1200. Las muestras se dividieron aleatoriamente en 6 grupos $(n=6)$ de acuerdo con los colorantes: agua destilada, cola, vino tinto, acai, café y cerveza oscura. El color de cada muestra se evaluó con un espectrofotómetro 3 veces: antes de blanquear (línea de base), después de blanquear con peróxido de hidrógeno al $35 \%$ durante 45 minutos y después de sumergirlo en bebidas colorantes. La medición del color se basó en la escala CIE $L{ }^{*} a{ }^{*} b$ *. Los datos se analizaron con ANOVA y Kruskal-Wallis, las comparaciones post-hoc se probaron como prueba de StudentNewman-Keuls y el nivel de significación fue $p<0,05$. Resultados: No hubo diferencia estadística entre los valores de los grupos de refrescos a base de cola $\Delta \mathrm{E}{ }^{*}$, vino tinto, jugo de acai, café y cerveza oscura ( $\mathrm{p}>$ 0.05). Conclusión: Los refrescos a base de cola, vino tinto, jugo de acai, café y cerveza oscura interfieren con la estabilidad del color del esmalte blanqueado con peróxido de hidrógeno al 35\%.

Palabras clave: Blanqueamiento dental, Peróxido de hidrógeno, Esmalte dental.

\section{INTRODUÇÃO}

Os dentes anteriores são fundamentais no estabelecimento do padrão estético do sorriso, no relacionamento social e na qualidade de vida das pessoas (ODIOSO LL, et al., 2000; PAVICIC DK, et al., 2019). Entretanto, aproximadamente, $1 / 3$ da população demonstrou algum tipo de insatisfação relacionada com a cor dos dentes (ODIOSO LL, et al., 2000). Os clareamentos dentários são amplamente empregados com a finalidade de recuperar a coloração natural dos dentes ou torná-los mais brancos (CAREY CM, 2014; EPPLE M, et al., 2019). A técnica de clareamento de dentes vitais realizada no consultório utiliza peróxidos de alta concentração e tem obtido resultados satisfatórios em avaliações laboratoriais e clínicas (DE GEUS JL, et al., 2016; LIMA DA, et al., 2015; MOUNIKA A, et al. 2018; PEREIRA SANCHEZ N, et al. 2017).

O sucesso dos protocolos de clareamento está diretamente relacionado a capacidade de difusão do peróxido de hidrogênio, o principal componente ativo dos agentes clareadores, que em contato com o dente se decompõe em dois subprodutos: água e oxigênio nascente (CAREY CM, 2014; KWON SR e WERTZ PW, 2015). Devido ao seu baixo peso molecular, o oxigênio apresenta alto poder de penetração e interage com os cromatóforos no interior da estrutura dentária (JOINER A e LUO W, 2007; KWON SR e WERTZ PW, 2015). Os cromóforos são compostos orgânicos que possuem longas cadeias contendo duplas ligações carbônicas, e o oxigênio promove a quebra destas ligações através de uma reação de oxidação, resultando na percepção de clareamento dos dentes (KWON SR e WERTZ PW, 2015). Contudo, o clareamento dentário pode causar alterações na ultraestrutura, composição química e microdureza do esmalte (VILHENA KFB, et al. 2019). Desta forma, ainda há uma controvérsia no meio científico se a realização de clareamento dentário pode facilitar a penetração de novos pigmentos, oriundos de alimentos ou bebidas, promovendo a recorrência do manchamento extrínseco (CÔRTES G, et al., 2013; KARADAS M e NILGUN S, 2014; NOGUEIRA JS, et al., 2019; ZHAO X, et al. 2019).

Muitas pessoas ao redor do mundo possuem o hábito de ingerir com frequência bebidas corantes e ácidas, como bebidas alcoólicas, cafés, sucos de frutas e refrigerantes (OZEN AE, et al, 2012; REZENDE M, et al., 2013). O café, o vinho e os refrigerantes a base de cola possuem colorações escuras, pH ácido e tem o potencial para causar manchamentos em dentes submetidos a clareamento (KARADAS M e NILGUN S, 2014; PIROLO R, et al., 2014; REZENDE M, et al., 2013). Por outro lado, o consumo de açaí é um hábito comum na região norte do Brasil e tem se difundido por outras regiões do país e do mundo (REBELO DE SOUSA K, 
et al. 2012). O açaí é uma bebida com coloração roxa escura, obtida a partir do fruto da planta do açaí, e é uma importante fonte de lipídios, proteínas, fibras, vitaminas B1 e E, ferro e antocianinas, que são os corantes naturais (AMARAL FR, et al., 2015). Estudos recentes têm demonstrado que 0 açaí tem um elevado potencial de manchamento do esmalte (REBELO DE SOUSA K, et al. 2012; AMARAL FR, et al., 2015)

A cerveja é a bebida alcoólica mais popular no mundo e a terceira mais consumida no planeta (ANTONOV M, et al. 2016; MARDEGAN SF, et al. 2013). No Brasil, um país tropical, o consumo de cerveja é considerado elevado chegando a aproximadamente 52 litros/ pessoa/ ano (NOGUEIRA FN, et al. 2000). A cerveja é o resultado de uma fermentação complexa de produtos amiláceos, como arroz, cevada e trigo, e pode apresentar diferentes colorações, variando entre o amarelo claro até o marrom escuro (MARDEGAN SF, et al. 2013; VANDERHAEGEN B, et al. 2007). Adicionalmente, algumas cervejas são ácidas, possuindo pH que variam entre 3,79 e 4,80, e apresentam potencial erosivo frente ao esmalte dentário (NOGUEIRA FN, et al. 2000; ZANATTA RF, et al., 20016). Embora seja uma bebida muito consumida, pouco se sabe sobre 0 potencial de machamento do esmalte clareado quando exposto a cervejas escuras e ácidas. Diante do exposto, o objetivo do presente estudo foi avaliar a influência de bebida corantes e ácidas na estabilidade de cor do esmalte submetido a clareamento com peróxido de hidrogênio à 35\%.

\section{MÉTODOS}

O presente estudo teve aprovação no Comitê de Ética de Pesquisa em Humanos (Protocolo № 2.758.761) e 20 terceiros molares humanos hígidos foram cortados com o auxílio de um disco diamantado (Extec Corp, Enfield, CT, EUA) para obter fragmentos de esmalte quadrangulares de de $4 \times 4 \mathrm{~mm}$. Os fragmentos foram embutidos (PRE30Mi; Arotec, São Paulo, SP, Brasil) em resina acrílica e posteriormente, planificados e polidos com lixas de carbeto de silício de granulações 320, 400, 600 e 1200 (Norton Abrasivos, São Paulo, SP, Brasil), acopladas em uma máquina politriz elétrica (Aropol 2V; Arotec, São Paulo, SP, Brasil). Cada etapa do processo de planificação e polimento foi realizada durante 60 segundos.

Os espécimes foram avaliados com uma lupa estereoscópica (StereoZoom Leica S8 APO, Leica Microsystems, Wetzlar, Alemanha) com 40 vezes de aumento, para garantir a presença de espécimes exclusivamente em esmalte, sem trincas e sem áreas de dentina exposta. Posteriormente, foram aleatoriamente divididos através do programa Excel (Excel 2013, Microsoft Corporatin, One Microsoft Way, Redmond, WA, EUA) em 6 grupos ( $n=6$ ), de acordo com as bebidas: Água destilada (controle), Refrigerante a base de cola, Vinho, Açaí, Café e Cerveja escura (Tabela 1).

Tabela 1 - Dados de fabricação e nível de pH das bebidas usadas nos grupos experimentais.

\begin{tabular}{lll}
\hline Grupo & Marca/fabricante & $\mathbf{p H}$ \\
\hline Água destilada (controle) & - & 7,57 \\
Refrigerante a base de cola & Coca Cola, Rio de Janeiro, RJ, Brasil. & 2,11 \\
Vinho & $\begin{array}{l}\text { Petirrojo; Bisquertt, Valle de Colchagua. } \\
\text { Chile. }\end{array}$ & 3,10 \\
Açaí & Frutã, Jaguaribe, CE, Brasil & 4,75 \\
Café & Nescafé, Nestle, Araras, SP, Brasil. & 5,03 \\
Cerveja escura & Ekaut Extra Stout; Cervejaria Ekaut, Recife, & 4,33 \\
& PE, Brasil. &
\end{tabular}

Fonte: Neri JR, et al., 2019. 
Inicialmente, a cor de cada espécime foi avaliada, com o auxílio de espectrofotômetro (VITA Easyshade Advance 4.0, Vident, Brea, CA, EUA), e registrada individualmente (baseline). Posteriormente, todos os espécimes foram submetidos ao protocolo de clareamento utilizando um agente clareador à base de peróxido de hidrogênio a 35\% (Whitness HP blue; FGM, Joinville, SC, Brasil - lote \#80172310072), durante 45 minutos. O protocolo de clareamento foi repetido em um total de 3 vezes, simulando o clareamento de consultório. Após 24 horas do clareamento, os espécimes foram novamente avaliados com o espectrofotômetro, e os dados foram individualmente registrados (intermediária), para verificar a efetividade do clareamento.

Após protocolo de clareamento, cada espécime foi submerso, individualmente, em $5 \mathrm{ml}$ de bebida a $37^{\circ}$ C, durante 6 horas em cada dia, até o total de 30 dias. Nos intervalos de imersão, as amostras foram mantidas em saliva artificial a $37^{\circ} \mathrm{C}$. (KARADAS M e NILGUN S, 2014). O café foi preparado, de acordo com as instruções de seu fabricante, na proporção de $8 \mathrm{mg}$ de café para $50 \mathrm{ml}$ de água morna. Para preparação do açaí, foi utilizado $100 \mathrm{~g}$ da polpa para $200 \mathrm{ml}$ de água gelada, conforme instruções do fabricante. Para prevenir possíveis alterações químicas nas bebidas, foram realizadas trocadas a cada 24 horas.

Após 30 dias, os espécimes foram removidos dos frascos contendo a bebida corante, e lavados com água destilada durante 10 segundos. Em seguida, foram, cuidadosamente, secos com um jato de ar livre de água e óleo, durante 10 segundos e então, foi realizada a avaliação final com espectrofotômetro de refletância, que realizou a mensuração da cor por meio de valores correspondentes à escala CIE L* $a^{*} b^{*}$. Nesse sistema, $L^{*}$ indica a luminosidade, e o $a^{*} e b^{*}$, o matiz, sendo que $o a^{*}$ representa a saturação no eixo vermelho verde $e$ $o b^{*}$ no eixo azul-amarelo.

A comparação da cor antes e após os espécimes serem submetidos às bebidas foi estabelecida pela diferença de cor ou $\Delta \mathrm{E}$, representada pela equação:

$\Delta E^{*} a b=\left[\left(\Delta L^{*}\right)^{2}+\left(\Delta a^{*}\right)^{2}+\left(\Delta b^{*}\right)^{2}\right] 0.5$, onde

$\Delta L^{*}=L^{*} 1-L^{*} 0$ (leitura após imersão nas bebidas menos leitura prévia à imersão nas bebidas)

$\Delta \mathrm{a}^{*}=\mathrm{a}^{*} 1-\mathrm{a}^{*} 0$ (leitura após imersão nas bebidas menos leitura prévia à imersão nas bebidas)

$\Delta b^{*}=b^{*} 1-b^{*} 0$ (leitura após imersão nas bebidas menos leitura prévia à imersão nas bebidas)

As tomadas de cor sempre foram realizadas no mesmo lugar do espécime através de uma demarcação realizada na primeira análise, guiando assim as leituras subsequentes, bem como o mesmo ambiente e mesma luminosidade, a fim de evitar que as futuras medidas fossem realizadas com diferenças em relação à luz. A ponteira de medição foi apoiada e assentada completamente, de forma perpendicular à superfície do esmalte.

Quando o valor de $\Delta \mathrm{E}^{\star}$ foi maior que 3,7 considerou-se uma variação de cor facilmente visível clinicamente. Valores entre 3,7 e 1, foram considerados como uma diferença de cor clinicamente aceitável. Por outro lado, valores de $\Delta \mathrm{E}^{*}$ menores que 1 foram considerados como clinicamente não perceptíveis (REIS RS, et al., 1996).

A análise estatística dos resultados foi realizada com o programa SigmaStat 3.5 (Systat Software Inc., San Jose, CA, EUA). Os valores referentes a $\Delta \mathrm{L}^{*}, \Delta \mathrm{a}^{*}, \Delta \mathrm{b}^{*} \Delta \mathrm{E}^{*} \mathrm{e}$ foram, inicialmente, avaliados com os testes de Shapiro-Wilk e Brown-Forsythe para analisar a distribuição normal dos dados e a igualdade de variância, respectivamente. Os dados paramétricos foram analisados com o teste de Análise de Variância e os dados não paramétricos foram submetidos ao teste de Kruskal-Wallis. Para as comparações post-roc foi utilizado o teste de Student-Newman-Keuls e o nível de significância adotado foi de $p<0,05$. As hipóteses nulas testadas foram que não haveria diferença estatística entre os grupos quanto aos valores de $\Delta \mathrm{L}^{*}, \Delta \mathrm{a}^{*}, \Delta \mathrm{b}^{*}$ e $\Delta \mathrm{E}^{*}$.

\section{RESULTADOS}

A efetividade do procedimento de clareamento com a média dos valores de $\Delta \mathrm{E}$ de todos os espécimes clareados $(\Delta \mathrm{E}=7,12 \pm 3,05)$. Após o clareamento, o valor de $\Delta \mathrm{L}$ foi positivo $(3,30 \pm 3,88)$ indicando que os 
espécimes ficaram mais claros. Por outro lado, os valores de $\Delta a(-1,25 \pm 2,17)$ e $\Delta b(-3,93 \pm 3,73)$ foram negativos, o que indica uma variação de cor dos espécimes para o espectro do verde e do azul, respectivamente.

Os dados referentes à avaliação dos parâmetros $\Delta \mathrm{L}^{*}, \Delta \mathrm{a}^{*}, \Delta \mathrm{b}^{*} \mathrm{e} \Delta \mathrm{E}^{*}$ estão na Tabela 2.

Tabela 2 - Média dos valores de $\Delta \mathrm{L}{ }^{*}, \Delta \mathrm{a}{ }^{*}, \Delta \mathrm{b}^{*}$ e $\Delta \mathrm{E}{ }^{*}$ do esmalte clareado exposto a diferentes bebidas corantes e ácidas.

\begin{tabular}{lcccc}
\hline Grupos $(\boldsymbol{n}=\mathbf{6})$ & $\mathbf{\Delta} \mathbf{L}^{\mathbf{*}}$ & $\mathbf{\Delta \mathbf { a } ^ { * }}$ & $\mathbf{\Delta} \mathbf{b}^{\mathbf{*}}$ & $\mathbf{\Delta} \mathbf{E}^{\boldsymbol{*}}$ \\
\hline Água destilada (controle) & $0,80 \pm 1,34^{\mathrm{A}}$ & $1,98 \pm 2,01^{\mathrm{A}}$ & $2,00 \pm 1,97^{\mathrm{AB}}$ & $2,68 \pm 1,17^{\mathrm{A}}$ \\
Refrigerante a base de cola & $-1,76 \pm 6,06^{\mathrm{B}}$ & $2,71 \pm 4,61^{\mathrm{A}}$ & $10,76 \pm 3,93^{\mathrm{A}}$ & $12,83 \pm 4,37^{\mathrm{B}}$ \\
Vinho & $-10,41 \pm 6,70^{\mathrm{C}}$ & $3,13 \pm 2,45^{\mathrm{A}}$ & $-5,43 \pm 9,15^{\mathrm{B}}$ & $14,82 \pm 6,83^{\mathrm{B}}$ \\
Açaí & $-11,3 \pm 2,77^{\mathrm{C}}$ & $3,93 \pm 0,49^{\mathrm{A}}$ & $3,95 \pm 6,25^{\mathrm{AB}}$ & $14,49 \pm 2,78^{\mathrm{B}}$ \\
Café & $-9,70 \pm 1,37^{\mathrm{C}}$ & $4,31 \pm 2,69^{\mathrm{A}}$ & $0,23 \pm 5,59^{\mathrm{AB}}$ & $12,43 \pm 4,43^{\mathrm{B}}$ \\
Cerveja escura & $-4,18 \pm 3,85^{\mathrm{D}}$ & $4,05 \pm 1,91^{\mathrm{A}}$ & $8,31 \pm 9,39^{\mathrm{A}}$ & $12,38 \pm 6,81^{\mathrm{B}}$
\end{tabular}

Legenda: * Letras maiúsculas semelhantes nas colunas indicam que não há diferença significativa entre os grupos $(p>0,05)$

Fonte: Neri JR, et al., 2019.

Análise dos valores de $\Delta \mathrm{L}^{*}$ (variação no brilho)

Os valores de $\Delta L^{*}$ foram significativamente influenciados pela exposição às bebidas corantes $(p<0,001$; $\mathrm{H}=20.823$ com 5 graus de liberdade). Todos os grupos apresentaram resultados negativos com exceção dos espécimes imersos em água destilada, o que indica que os espécimes ficaram mais escuros após a exposição às bebidas. Não houve diferença estatística entre os espécimes expostos ao vinho tinto, ao café e ao açaí $(p>0,05)$, e estes espécimes apresentaram os resultados de $\Delta L^{*}$ mais baixos $(p<0,05)$. O maior valor de $\Delta L^{*}$ foi observado nos espécimes expostos à água destilada, seguidos do refrigerante a base de cola e da cerveja escura $(p<0,05)$.

Análise dos valores de $\Delta \mathrm{a}^{*}$ (variação eixo vermelho-verde)

Os valores de $\Delta a^{*}$ não foram significativamente influenciados pela exposição às bebidas corantes $(p=0,741 ; F=0.544)$. Não houve diferença estatística entre os valores de $\Delta a^{*}$ de todos os grupos testados $(p>0,05)$. Todos os grupos apresentaram resultados positivos de $\Delta a^{*}$, o que indica uma mudança do espectro de cor dos espécimes para o vermelho.

Análise dos valores de $\Delta b^{*}$ (variação no eixo amarelo-azul)

Os valores de $\Delta b^{*}$ foram significativamente influenciados pela exposição às bebidas corantes $(p=0,004$; $\mathrm{F}=4.502$ ). Os espécimes expostos ao vinho apresentaram valores $\Delta \mathrm{b}^{*}$ negativos, indicado uma mudança de cor para o espectro azul. Não houve diferença estatística entre os valores de $\Delta b^{*}$ dos grupos Refrigerante a base de cola e Cerveja escura ( $p>0,05)$, e estes foram estatisticamente maiores do que o grupo Vinho tinto $(p<0,05)$. Não houve diferença estatística entre os grupos Água destilada, Açaí e Café e o Vinho tinto $(p>0,05)$.

Análise dos valores de $\Delta \mathrm{E}^{*}$ (total da variação de cor)

Os valores de $\Delta \mathrm{E}^{*}$ foram significativamente influenciados pela exposição às bebidas corantes $(p=0,002$; $\mathrm{F}=5.158$ ). Os menores valores de $\Delta \mathrm{E}^{*}$ foram observados no grupo Água destilada $(p<0,05)$. Não houve 
diferença estatística entre os valores de $\Delta \mathrm{E}^{*}$ grupos Refrigerante a base de cola, Vinho tinto, Açaí, Café e Cerveja escura $(p>0,05)$, e todos foram superiores a 3,7 , portanto visíveis clinicamente.

\section{DISCUSSÃO}

A avaliação da estabilidade de cor dos dentes pode ser realizada através de métodos subjetivos ou objetivos (KARADAS M e NILGUN S, 2014; REZENDE M, et al. 2013). O uso de colorimetria computadorizada ou a espectrofotometria com base nos parâmetros do CIE-Lab (1971) (Commission Internationale d'Eclairage, $\mathrm{L}=$ luminosidade, $\mathrm{a}=$ croma no eixo vermelho-verde, $\mathrm{b}=$ croma no eixo amarelo-azul) permite uma comparação matemática e obtêm dados mais precisos e consistentes (SEGHI RR, et al., 1989). A espectrofotometria é o método de avaliação de cor de estruturas dentais mais difundidos no meio científico (AMARAL FR, et al. 2015; CARLOS NR, et al., 2019; KARADAS M e NILGUN S, 2014; PIROLO R, et al. 2014). Devido às razões acima citadas, a espectrofotometria foi utilizada para as análises do presente estudo.

A exposição de esmalte clareado a bebidas corantes e ácidas promove uma redução significativa no brilho $\left(\Delta L^{*}\right)$ (AMARAL FR, et al., 2015; KARADAS M e NILGUN S, 2014; PIROLO R, et al. 2014), estes dados corroboram com os achados do presente estudo e, desta forma, a primeira hipótese nula foi rejeitada. $O$ vinho possui a capacidade de reduzir a luminosidade dos dentes, após 15 dias de exposição. Amaral FR, et al. (2015) observaram que o $\Delta L^{*}$ do esmalte foi significativamente menor quando imersos em açaí, durante 28 dias, em comparação ao café. Enquanto Pirolo R, et al. (2014) verificaram que não houve diferença estatística entre os valores de brilho do esmalte exposto ao café e ao refrigerante a base de cola, após 3 dias de imersão, e ambos tornaram esse substrato mais escuro.

Substâncias corantes podem promover alterações no $\Delta$ a dos substratos dentários, o componente que avalia a variação de cor entre os espectros vermelho e verde (CARLOS NR, et al., 2019; KARADAS M e NILGUN S, 2014; ZHAO X, et al., 2019). O vinho e o café promovem uma elevação considerável no $\Delta$ a, tornando os dentes mais avermelhados (ZHAO X, et al., 2019). Carlos NR, et al. (2019) indicaram que o esmalte clareado adsorveu pigmentos avermelhados quando exposto ao refrigerante a base de cola e ao café. O açaí quando em contato com o esmalte clareado tornou o substrato significativamente mais avermelhado, do que o café (AMARAL FR, et al., 2015). No presente estudo, todas as bebidas corantes e ácidas tornaram o esmalte clareado mais vermelhos, porém, não houve diferença estatística entre os grupos. Portanto, a segunda hipótese nula falhou em ser rejeitada.

$\mathrm{O}$ espectro de cores mais importante para o clareamento dentário está relacionado ao $\Delta \mathrm{b}$, pois corresponde à variação de cor entre o amarelo (valores positivos) e o azul (valores negativos), e este componente parece ser afetado por alimentos e bebidas corantes (AMARAL FR, et al., 2015; CARLOS NR, et al., 2019; KARADAS M e NILGUN S, 2014). AMARAL FR, et al. (2015) verificaram que o café promoveu uma significativa variação de cor do esmalte clareado para o espectro amarelo, enquanto o açaí tendeu ao azul. A exposição do esmalte clareado a refrigerantes a base de cola os torna consideravelmente mais amarelados quando comparado ao café (CARLOS NR, et al., 2019). No presente estudo, houve uma diferença significativa entre os valores de $\Delta b$ entre o vinho e a cerveja escura, onde os valores do vinho foram negativos, demonstrando evidente mudança de cor do esmalte clareado para o azul, e a cerveja escuro variou a coloração para o amarelo. Desta forma, a terceira hipótese nula foi rejeitada.

A ingestão frequente de alguns alimentos e bebidas podem causar manchamento dentário (REBELO DE SOUSA K, et al., 2012). O manchamento dental pode ser influenciado por diferentes fatores, dentre eles 0 baixo $\mathrm{pH}$ de bebidas corantes (ZANATTA RF, et al., 20016). $\mathrm{O} \mathrm{pH}$ ácido destas bebidas solubiliza os prismas de esmalte, aumenta a permeabilidade e a penetração de substâncias corantes no interior da estrutura dentária submetidas ao clareamento (CARLOS NR, et al., 2019; EHLEN LA, et al., 2008; KARADAS M e NILGUN S, 2014; NOGUEIRA FN, et al., 2000). De acordo com alguns estudos (KARADAS M e NILGUN S, 2014; PIROLO R, et al. 2014; SILVA LP, et al., 2013), a exposição de dentes clareados a refrigerantes à base de cola, uma bebida ácida, interferiu significativamente na estabilidade de cor $\left(\Delta \mathrm{E}^{*}\right)$, corroborando com os resultados do presente estudo. Portanto, a quarta hipótese nula foi rejeitada. 
O café é uma bebida de baixo $\mathrm{pH}$ que possui alta concentração de íons metálicos, como potássio, magnésio, ferro e manganês, e concentrações menores de estrôncio, zinco, níquel e cádmio (MANNO SHC, et al. 2018). A exposição do esmalte ao café resultou em sequestro de cálcio e fosfato da estrutura dentária e aumentou a concentração de magnésio, ferro e potássio, levando a uma considerável alteração de cor (PRSKALO K, et al. 2017; MANNO SHC, et al. 2018). Por outro lado, alguns trabalhos in vitro observaram que o café promoveu uma alteração de cor no esmalte clareado significativamente menor do que o refrigerante a base de cola (KARADAS M e NILGUN S, 2014; PIROLO R, et al. 2014). No presente estudo, não houve diferença estatística entre os grupos café e refrigerante a base de cola, quanto a variação de cor. Este resultado pode ter relação com a preparação do café, seguindo as recomendações do fabricante, que resultou em uma solução mais concentrada do que a formulação dos demais estudos e, provavelmente, pode ter influenciado na variação de cor. Novos estudos devem ser conduzidos para avaliar se a concentração do café pode interferir significativamente na variação de cor do esmalte submetido a clareamento.

$\mathrm{O}$ vinho tinto contém uma grande quantidade de pigmentos, além de um baixo $\mathrm{pH}$, o que intensifica a desmineralização da superfície do esmalte e o torna mais vulnerável ao manchamento (BERGER SB, et al., 2008; PIROLO R, et al. 2014; ZHAO X, et al. 2019). Adicionalmente, o clareamento aumenta a suscetibilidade de manchamento do esmalte dentário pelo vinho (BERGER SB, et al., 2008), estes dados dão suporte aos achados do presente estudo. Por outro lado, Liporoni PC, et al (2010) observaram que o vinho manchou o esmalte clareado, após 30 e 150 minutos de exposição, enquanto o café não interferiu no processo. Karadas $M$ e Nilgun $S$ (2014) verificaram que os valores de $\Delta E$ do esmalte clareado exposto ao vinho foram significativamente menores do que os do refrigerante a base de cola. Estes dados contrastam com os resultados do presente estudo, onde não houve diferenças entre os valores de $\Delta \mathrm{E}$ do esmalte clareado $\mathrm{e}$ exposto ao vinho e refrigerante a base de cola. É possível sugerir que os valores aproximados do $\mathrm{pH}$ do vinho $(3,10)$ e do refrigerante a base de cola $(2,11)$, podem ter causado padrões erosivos similares e resultou alterações de cor semelhantes.

Existem relatos indicando que o açaí promove manchamento nos substratos dentários (AMARAL FR, et

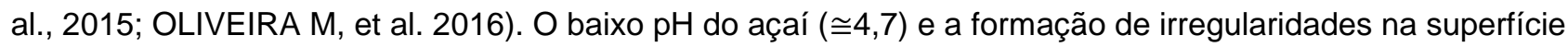
do esmalte, podem ser considerados fatores que contribuem para o padrão de coloração encontrado no presente estudo. Além disso, o açaí é um dos produtos mais ricos em um corante natural do grupo dos polifenóis chamado antocianina e que possui alto poder de pigmentação (AMARAL FR, et al., 2015). Amaral FR, et al (2015) observaram que o esmalte clareado é mais suscetível ao manchamento pelo açaí do que pelo café, após 7 dias de exposição. O tempo de exposição é um parâmetro que parece interferir do grau de pigmentação dos substratos dentários (KARADAS M e NILGUN S, 2014). Desta forma, no presente estudo o café teve um poder de coloração maior, provavelmente, devido ao tempo mais prolongado de exposição do esmalte clareado (30 dias).

As cervejas têm potencial de reduzir a microdureza e promover a dissolução do esmalte dentário (NOGUEIRA FN, et al. 2000; ZANATTA RF, et al., 20016). Adicionalmente, Antonov M, et al. (2016) evidenciaram que a exposição de resinas compostas a diferentes cervejas promoveu significativa alteração de $\Delta \mathrm{E}$. A intensidade do manchamento está associado a composição, ao potencial de adsorção das cervejas e o tempo de exposição. Cervejas escuras, do tipo stout, possuem alta concentração de proteínas, aminoácidos aromáticos (triptofano, tirosina e fenilalanina), riboflavina e compostos fenólicos e apresentam maior potencial de pigmentação (ANTONOV M, et al. 2016; DRAMIĆANIN T, et al. 2019). Portanto, estes dados dão suporte aos resultados do presente estudo.

\section{CONCLUSÃO}

As bebidas ácidas e com elevada concentração de corantes, como refrigerantes à base de cola, vinho tinto, açaí, café e cerveja escura, interferem significativamente na estabilidade da cor do esmalte clareado, com produtos à base de peróxido de hidrogênio a $35 \%$. A associação entre agentes clareadores e bebidas com baixo $\mathrm{pH}$ pode causar alterações na ultraestrutura e composição química do esmalte. Neste contexto, os prismas de esmalte são solubilizados e há uma considerável elevação da permeabilidade do substrato a 
penetração de pigmentos oriundos de alimentos ou bebidas. Portanto, o hábito de ingerir bebidas corantes e ácidas com grande frequência pode ser um fator limitante na longevidade dos resultados decorrentes dos procedimentos clareadores.

\section{ACRÔNIMOS E ABREVIAÇÕES}

$\Delta L^{*}$ : delta $L$ (variação no brilho)

$\Delta \mathrm{a}^{*}$ : delta a (variação eixo vermelho-verde)

$\Delta \mathrm{b}^{*}$ : delta $\mathrm{b}$ (variação no eixo amarelo-azul)

$\Delta \mathrm{E}^{*}$ : delta $\mathrm{E}$ (total da variação de cor)

pH: potencial hidrogeniônico

№: número

$\mathrm{CT}$ : Connecticut

WA: Washington

CA: Califórnia

\section{REFERÊNCIAS}

1. AMARAL FR, et al. Effect of hydrogen peroxide in different concentrations on the degree of bleaching and susceptibility to staining. Scientific Journal of Dentistry, 2015; 2(3): 8-12.

2. ANTONOV M, et al. Changes of color and fluorescence of resin composites immersed in beer. Journal $f$ Esthetic and Restorative Dentistry, 2016; 28(5): 330-338.

3. BERGER SB, et al. Enamel susceptibility to red wine staining after $35 \%$ hydrogen peroxide bleaching. J Appl Oral Sci. 2008; 16(3): 201-214.

4. CAREY CM. Tooth Whitening: What We Now Know. J Evid Based Dent Pract. 2014; 14 Suppl: 70-76.

5. CARLOS NR, et al. Influence of Staining Solutions on Color Change and Enamel Surface Properties During At-home and In-office Dental Bleaching: An In Situ Study. Oper Dent. 2019; 44(6): 595-608.

6. CIE (Commission Internationale de l'Eclairage) (1971). Colorimetry, official recommendations of the International Commission on Illumination. Publication CIE No. 15 (E-1.3.1). Paris, France: Bureau Central de la CIE, 4 Av. du Recteur Poincaré, 75782 Paris Cedex 16.

7. CÔRTES G, et al. Influence of coffee and red wine on tooth color during and after bleaching. Acta Odontologica Scandinavica, 2013; 71(6): 1475-1480.

8. DE GEUS JL, et al. At-home vs In-office Bleaching: A Systematic Review and Meta-analysis. Oper Dent. 2016; 41(4): 341-356.

9. DRAMIĆANIN T, et al. The Parallel Factor Analysis of Beer Fluorescence. J Fluoresc. 2019; 29(5): 1103-1111.

10. EHLEN LA, et al. Acidic beverages increase the risk of in vitro tooth erosion. Nutr Res. 2008 May;28(5):299-303.

11. EPPLE M, et al. A Critical Review of Modern Concepts for Teeth Whitening. Dent J (Basel), 2019 Sep; 7(3): 79.

12. JOINER A, LUO W. Tooth colour and whiteness: A review. J Dent. 2017; 67S: S3-S10.

13. KARADAS M, NILGUN S. The effect of different drinks on tooth color after home bleaching. European journal of dentistry, 2014; 8(2): 249-253.

14. KWON SR, WERTZ PW. Review of the Mechanism of Tooth Whitening. J Esthet Restor Dent. 2015; $27(5): 240-257$.

15. LIMA DA, et al. In vitro effects of hydrogen peroxide combined with different activators for the in-office bleaching technique on enamel. Acta Odontol Scand. 2015; 73(7): 516-21.

16. LIPORONI PC, et al. Enamel susceptibility to coffee and red wine staining at different intervals elapsed from bleaching: a photoreflectance spectrophotometry analysis. Photomedicine and laser surgery, 2010; 28. S2: S-105.

17. MANNO SHC, et al. Spectroscopic examination of enamel staining by coffee indicates dentin erosion by sequestration of elements. Talanta. 2018; 189 (1): 550-559.

18. MARDEGAN SF, et al. Stable carbon isotopic composition of Brazilian beers-A comparison between large- and smallscale breweries. Journal of Food Composition and Analysis 2013; 29(1): 52-57. 
19. MOUNIKA A, et al. Clinical evaluation of color change and tooth sensitivity with in-office and home bleaching treatments. Indian J Dent Res. 2018; 29(4): 423-427.

20. NOGUEIRA FN, et al. In vitro approach to evaluate potential harmful effects of beer on teeth. J Dent. 2000; 28(4): 271 276.

21. NOGUEIRA JS, et al. Does consumption of staining drinks compromise the result of tooth whitening? J Clin Exp Dent. 2019; 11(11): e1012-e1017.

22. ODIOSO LL, et al. Impact of demographic, behavioral, and dental care utilization parameters on tooth color and personal satisfaction. Compend Contin Educ Dent Suppl. 2000; (29): S35-41.

23. OLIVEIRA M, et al. Optical Dental Whitening Efficacy of Blue Covarine Toothpaste in Teeth Stained by Different Colors. J Esthet Restor Dent. 2016; 28 Suppl 1: S68-77.

24. OZEN AE, et al. Worldwide consumption of functional foods: a systematic review. Nutrition Reviews. 2012; 70(8): 472481.

25. PAUL S, et al. Visual and spectrophotometric shade analysis of human teeth. J Dent Res. 2002;81(8):578-582.

26. PAVICIC DK, et al. Changes in quality of life induced by tooth whitening are not influenced by global self-esteem: a randomized double-blind placebo-controlled trial. Odontology, 2019; 1-9.

27. PEREIRA SANCHEZ N, et al. Whitening-dependent changes of fluorescence of extracted human teeth. J Esthet Restor Dent. 2017; 29(5): 352-355.

28. PIROLO R, et al. Effect of coffee and a cola-based soft drink on the color stability of bleached bovine incisors considering the time elapsed after bleaching. J Appl Oral Sci. 2014; 22(6): 534-540.

29. PRSKALO K, et al. Risk factors associated with black tooth stain. Acta Clin Croat 2017; 56(1): 28-35.

30. REIS RS, et al. Effect of firing on the color stability of a light-cured ceramic stain. J Prosthodont, 1996; 5(3): 182-185

31. REBELO DE SOUSA K, et al., Extrinsic tooth enamel color changes and their relationship with the quality of water consumed. Int J Environ Res Public Health. 2012; 9(10): 3530-3539.

32. REZENDE M, et al. Clinical effects of exposure to coffee during at-home vital bleaching. Oper Dent. 2013; 38(6): E22936.

33. SEGHI RR, et al. Performance assessment of colorimetric devices on dental porcelains. J Dent Res. 1989; 68(12): 1755-1759.

34. SILVA LP, et al. Evaluation of tooth bleaching effectiveness through an experimental biological model at Gallus spp. Eggshell. Braz Dent Sci. 2013; 16(4): 55-64.

35. VAN DER BURGT TP, et al. A comparison of new and conventional methods for quantification of tooth color. J Prosthet Dent. 1990; 63(2): 155-162.

36. VANDERHAEGEN B, et al. Aging characteristics of different beer types. Food Chemistry. 2007; 103(2): 404-412.

37. VILHENA KFB, et al. Dental enamel bleached for a prolonged and excessive time: Morphological changes. PLoS One. 2019; 14(4): e0214948.

38. ZANATTA RF, et al. Harmful Effect of Beer on Bovine Enamel Microhardness - In Vitro Study. PLoS One. 2016; 11(10): e0163440.

39. ZHAO X, et al. Effects of different discoloration challenges and whitening treatments on dental hard tissues and composite resin restorations. J Dent. 2019; 89(1): 103182. 